\title{
Room temperature Monte Carlo study of the mechanical properties of thermoplastic polymers
}

\author{
Silvio A. Ospina ${ }^{1}$, Michael Hess ${ }^{2}$, Betty L. López ${ }^{1}$ * \\ ${ }^{1}$ Grupo Ciencia de los Materiales, Instituto de Química, Universidad de Antioquia, \\ A.A: 1226, Medellín, Colombia; Fax 57-4-2330120; blopez@quimbaya.udea.edu.co \\ ${ }^{2}$ Department of Physical Chemistry, Gerhard Mercator University, 47048 Duisburg, \\ Germany; hi259he@uni-duisburg.de
}

(Received: April 14, 2004; published: April 30, 2004)

This work has been presented at the $11^{\text {th }}$ Annual POLYCHAR World Forum on Advanced Materials, January 7-10, 2003, in Denton, Texas, USA

\begin{abstract}
The effect of the tensile test rate upon the mechanical properties of semicrystalline high-density polyethylene (HDPE) is studied by the Monte Carlo method. A two-dimensional lattice model is used, which considers first and second neighbour's interactions between $\mathrm{CH}_{2}-\mathrm{CH}_{2}$ groups. Metropolis dynamics is implemented as energy minimization tool. Results reveal the existence of two distinguishable regions in the stress-strain curves. One of them is characterized by an elastic and linear behaviour below $0.27 \%$ of deformation where the elastic modulus is practically insensitive to the number of Monte Carlo steps, whereas at higher deformation the system exhibits a non-linear behaviour ascribed to the viscoelastic character of the material endorsed by a clear dependence of the relaxation modulus as a function of the strain rate. The relaxation behaviour of HDPE obtained in our simulation shows an exponential decrease of the stress as time increases, which agrees with experimental data.
\end{abstract}

\section{Introduction}

The physical and chemical properties of polymers have been of long standing interest for both scientists and engineers since the beginning of polymers research. Most of the earliest works on polymers dealt with macroscopic aspects from which it became clear that a deeper understanding could only be obtained on the basis of a molecular analysis [1].

The mechanical behaviour of polymers is still not well understood, and it is strongly dependent on many variables like the chemical nature, microstructure, morphology and external conditions. On the other hand, traction measurements are widely employed to evaluate the mechanical properties and to select a material for a specific application. Hence, properties like stress $(\sigma)$ and modulus $(E)$ at different stages of deformation are obtained. Moreover, polymeric materials show a time dependent viscoelastic behaviour, which is related to the stress relaxation at constant deformation $(\varepsilon)$ and can be represented [2] by 
$\sigma(t)_{\varepsilon}=E \varepsilon_{0} \mathrm{e}^{-t / t^{*}}$

where $\varepsilon_{0}$ is a constant corresponding to the initially applied strain, $E$ Young's modulus of the Hookean behaviour and $t^{*}$ the relaxation time. The time-dependent Young's modulus is usually called the tensile relaxation modulus, defined [2] as

$E(t)=\frac{\sigma(t)}{\varepsilon}$

When the mechanical force is applied, relaxational processes take place in a polymeric material: energy transmission across the chain and to the neighbouring chains by segmental motions and conformational rearrangements; bond stretching and angle changes leading to elastic energy storage; and phase transformations [3].

Increasing computer power has made simulation of mechanical properties popular. Most of these studies use molecular dynamics (MD) and Monte Carlo (MC) simulations [4-8]. In particular, Monte Carlo studies of polymers usually employ the bondfluctuation model developed by Carmesin and Kremer [1] as a lattice algorithm for two-dimensional polymer chains. Lattice-based models where polymer chains are represented by bonds on a grid have also been used by many workers to simulate the static and dynamic properties of polymer systems [9-12].

In this work we study the first steps of deformation of a high-density polyethylene exposed to an external applied force. The study is carried out with the framework of a 2D lattice model and the atomic Monte Carlo method as energy minimization tool, $\sigma(\varepsilon)$ and $E(t)$ curves are also computed and discussed.

\section{Simulation and theory}

The polymer chains of HDPE have been simulated through self-avoiding and growing random walks on a two-dimensional lattice with the origin at zero and the lattice coordinates from $-L \ldots+L$ so that the total lattice has $(2 L+1)^{2}$ lattice sites with periodic boundary conditions as it has been described elsewhere [13]. The following Hamiltonian has been used to describe the interactions between the $\mathrm{CH}_{2}$ groups $[14,15]:$

$H=-J \sum_{j=1}^{n}\left(1-\delta_{S i S j}\right)-\mathfrak{T} \sum_{i} \Delta x_{i}$

where

$\delta_{S i S j}=\left\{\begin{array}{l}0, \ldots . S i \neq S j \\ 1, \ldots S i=S j\end{array}\right\}$

The first term in Eq. (3) gives the molecular interaction of each $\mathrm{CH}_{2}$ group with the first and second topological nearest neighbours. We assume the same mean values of the cohesion energy $J$ of $50 \mathrm{meV}$ of the $\mathrm{CH}_{2}-\mathrm{CH}_{2}$ interactions $[16,17]$. The second term accounts for the interaction of each molecule with the external traction force $\mathfrak{I}$ applied along the $x$-axis. The position operator $\delta$ considers the position of each $\mathrm{CH}_{2}$ (Si) with respect to the nearest and next nearest neighbours ( $S j)$, and $n$ indicates the total number of these neighbours. Concerning the dynamics of the system we have implemented such different types of movements that the $\mathrm{CH}_{2}-\mathrm{CH}_{2}$ bond length remains constant according to the expected behaviour during the first deformation steps. In the employed Metropolis dynamics [18,11], the probability $W$ for a confor- 
mational change from the state $\mu$ to the state $v$ depends on the energy difference $\Delta E$ according to:

$W(\mu \rightarrow v)=\left\{\begin{array}{cc}1 & , \text { if } \Delta E \leq 0 \\ \exp \left(-\Delta E / k_{\mathrm{B}} T\right), & \text { if } \Delta E>0\end{array}\right.$

In this dynamics a Monte Carlo step (mcs) is defined as the elapsed time to visit all the $\mathrm{CH}_{2}$ groups in each chain and to attempt a movement to reach a new position in such a way that the excluded volume condition is obeyed. A Monte Carlo step can be considered proportional to the inverse of the test rate. The following quantities are computed: a) elastic modulus $\left(E_{0}\right)$, b) stress relaxation modulus $E(t)$ at different deformation percentages, and $c$ ) the average value $\langle\Delta x\rangle$ of the projection of the chains along the direction of the external applied force and consequently the percentage of deformation according to:

$\varepsilon=\frac{\langle\Delta x\rangle-\langle\Delta x\rangle_{0}}{\langle\Delta x\rangle_{0}} \times 100 \%$

where $\langle\Delta x\rangle_{0}$ is the average projection corresponding to the initial configuration of the system. Additionally, in order to simulate a semicrystalline polymer characterized by a high degree of extension (see Fig. 1), the chains in our polymer were preferentially oriented, with an average angle of $45^{\circ}$ of the direction with respect to the $x$-axis (direction of deformation) computed from the Hermans function [19], as the initial configuration state. Results presented in this work correspond to a system of 1200 chains inscribed on a lattice with $L=150$. The total number of $\mathrm{CH}_{2}$ groups is adjusted in such a way that the fraction of occupied sites (filling concentration) is $55 \%$. This value is a compromise between two requirements: it must be high enough in order to represent the typical properties of a dense polymer and on the other hand, it must be low enough in order to allow the movement of $\mathrm{CH}_{2}$ units during the deformation dynamics in a reasonable computing time [20]. Afterwards, the corresponding curves of stress (external applied force) as a function of deformation and for a different number of Monte Carlo steps are thus obtained.

\section{Results and discussion}

As an example, Fig. 1 shows a chain before and after applying the uniaxial external force $\mathfrak{I}$. This figure shows the way a typical HDPE chain is placed on the lattice and the effect of the external applied force for which a rotation and a small displacement of the chain is evidenced.

In order to evaluate the reproducibility of our model, several initial chain configurations have been considered changing the seed of the random number generator, which allows calculation of configurational average and error bars. Fig. 2 shows the simulation results for the HDPE at room temperature for different number of samples during the first deformation steps $(\varepsilon<1 \%)$. As it can be observed the reproducibility is quite good for a number of samples greater than 100 and the obtained error bars are very small (of the order of the symbol size). In the following always more than 500 samples were considered.

According to the standard test method for tensile properties of plastics, ASTM D63899 [21], a zero adjust for the deformation must be carried out by extrapolation of the straight line CD (Fig. 3(a)) to the deformation axis (point B). In our simulation, this 
point corresponds approximately to $0.28 \%$ and we assigned the zero value to this point (Fig. 3(b)). It must be pointed out that the obtained stress-strain dependence is in agreement to what is expected in real experiment.

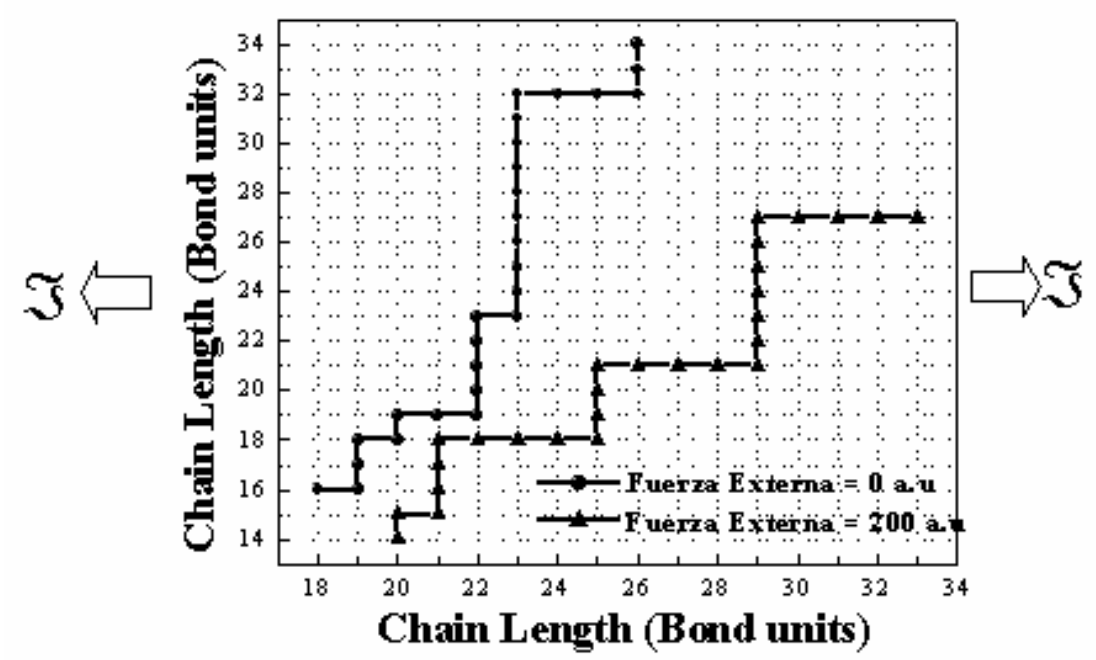

Fig. 1. Chain movement during deformation of the system

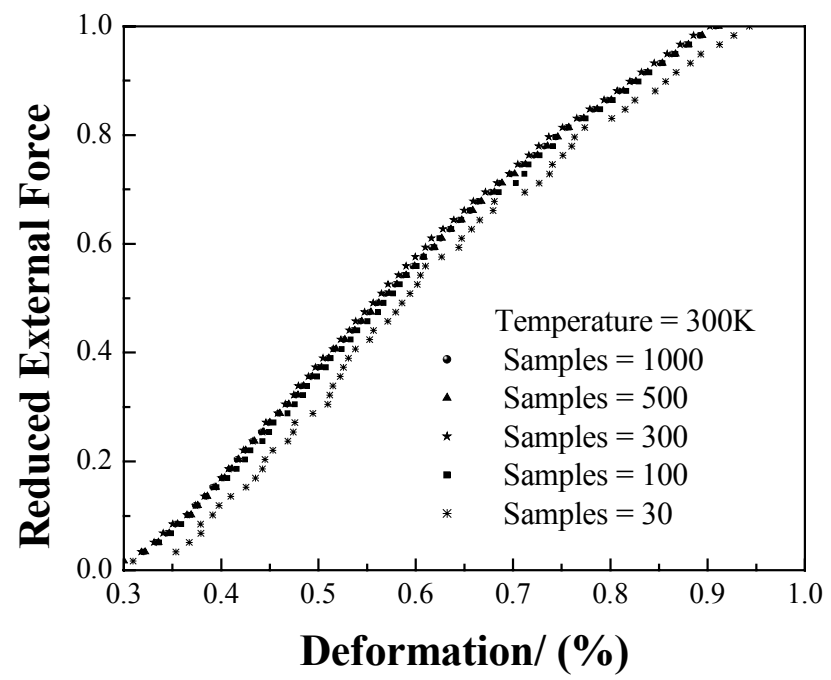

Fig. 2. Evaluation of the reproducibility of the simulation

Up to $\varepsilon \cong 0.27 \%$, region I Fig. 4 , all deformation curves practically coincide independently of the number of Monte Carlo steps or deformation rate. This behaviour is ascribed to the elastic response of the material, only valid for a very small deformation range. For the following stages after this elastic limit $(\varepsilon>0.27 \%$, region II), $E(t)$ varies with the deformation rate as can be deduced from the changes of slope with time. This fact is attributed to the viscoelastic behaviour of the material and is distinguished by an increase of the stress with the deformation rate at a given deformation, depending on the rigidity of the material. Thus, at high deformation rates (one or two Monte Carlo steps), the system behaves in a more rigid way and the chains tend to stay on their original positions, creating a smaller percentage of deformation and a higher stress in such a way that the elastic behaviour is preserved over a wider range. Beyond two Monte Carlo steps, the polymer has enough time to respond to the actions of stress and deformation. The linear elastic region diminishes 
with the increment of time favouring the relaxation and energy dissipation, which is reflected in a smaller elastic modulus.
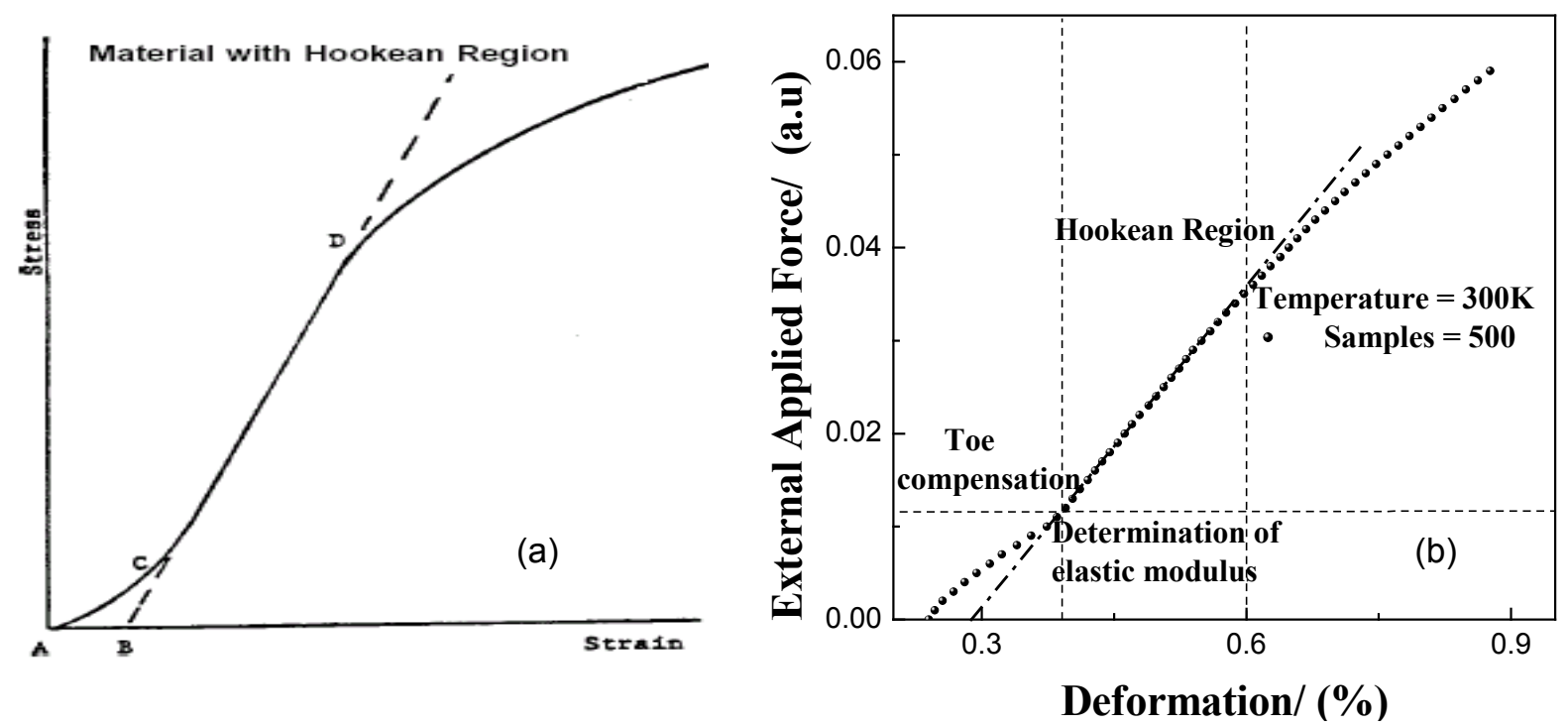

Fig. 3. Stress vs. deformation graphs: (a) Plastic material (Hookean Region) [21]; (b) MC simulation of PE

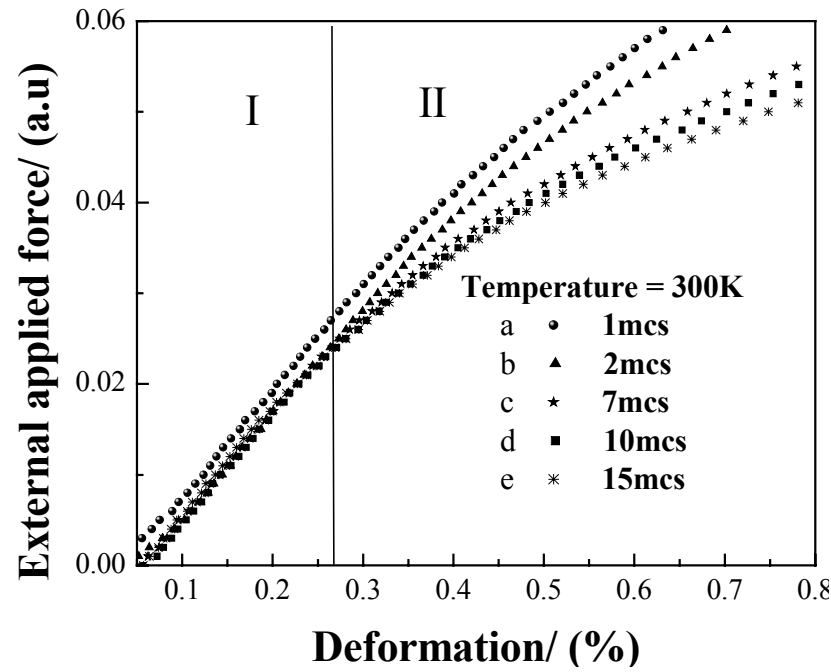

Fig. 4. MC simulation results for PE with Hookean region

The relaxation modulus as a function of time at room temperature $\left(27^{\circ} \mathrm{C}\right)$ at several deformations is shown in Fig. 5(a,b) from which a decrease of the relaxation modulus is remarkable at low percentage of deformation $(\varepsilon \approx 0.5 \%)$, obeying a non-linear viscoelastic behaviour [22]. The relaxation modulus acquires a constant value at high rates (which is equivalent to a large number of mcs) and deformations higher than $3 \%$. The estimation of average relaxation time at $4 \%$ is obtained from the inverse of the slope of Fig. 6(b) and correlated with an experimental result [23] giving a relaxation time of $\mathrm{mcs} \approx 70 \mathrm{~s}$.

Fig. 6 shows experimental and MC-simulated reduced stress relaxation vs. time, obtained from the stress-strain simulated curves at different deformation rates. The relaxation behaviour of HDPE obtained in our simulation shows an exponential 
decrease of the stress as time increases according to Eq. (1), which agrees with experimental data at short relation times [23] and there is a deviation of the experimental data for times greater than $415 \mathrm{~s}$, which could be due to the fact that the simulation does not consider ramification, nor any entanglement of the chains, and PE has low molecular weight compared with real HDPE, also the simulation is carried out on a 2D lattice and not on the real 3D structure. It is also noticed that at long times a nonzero stress level is gradually attained (incomplete relaxation). This stress level approached after sufficiently long measuring times is often referred to as the internal stress level [3], which has been already reported in refs. [2,23].

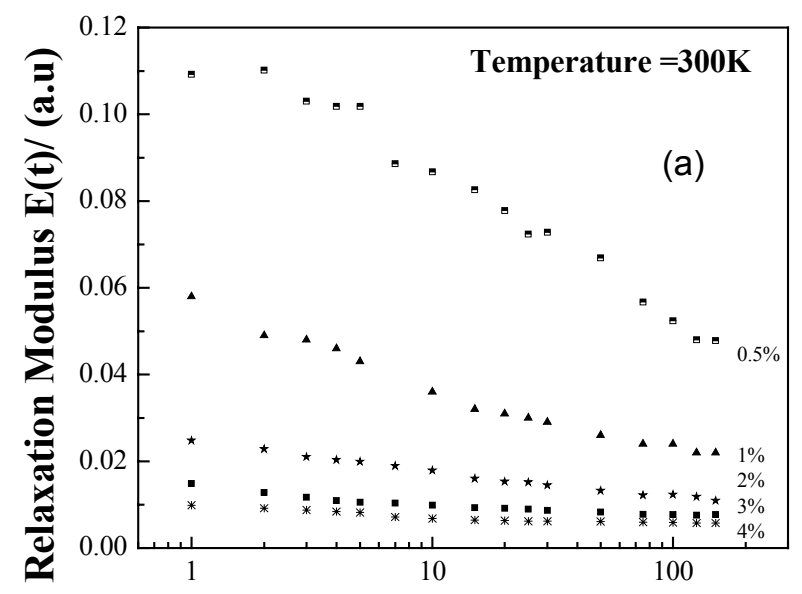

Deformation Time/ (mes)

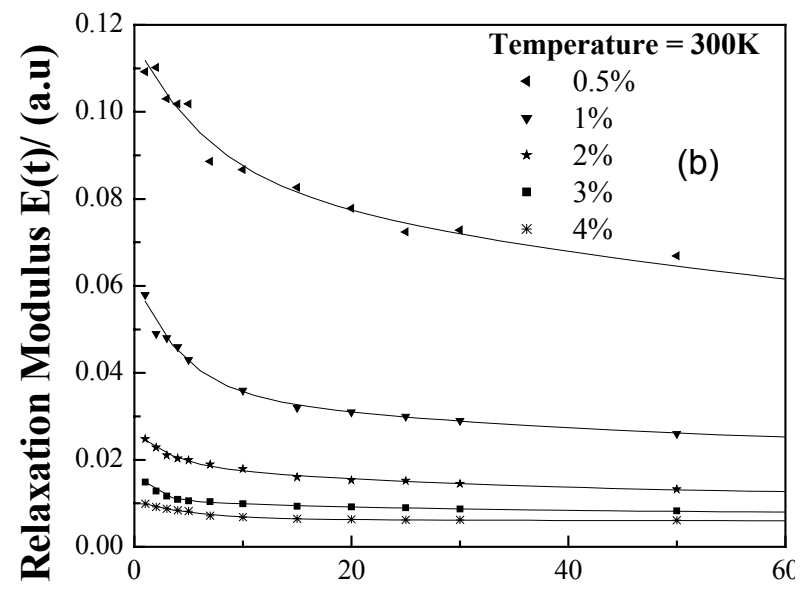

Deformation Time/ (mes)

Fig. 5. MC simulation result for PE: nonlinear viscoelastic relaxation modulus of PE: (a) linear scale, (b) semi-logarithmic scale

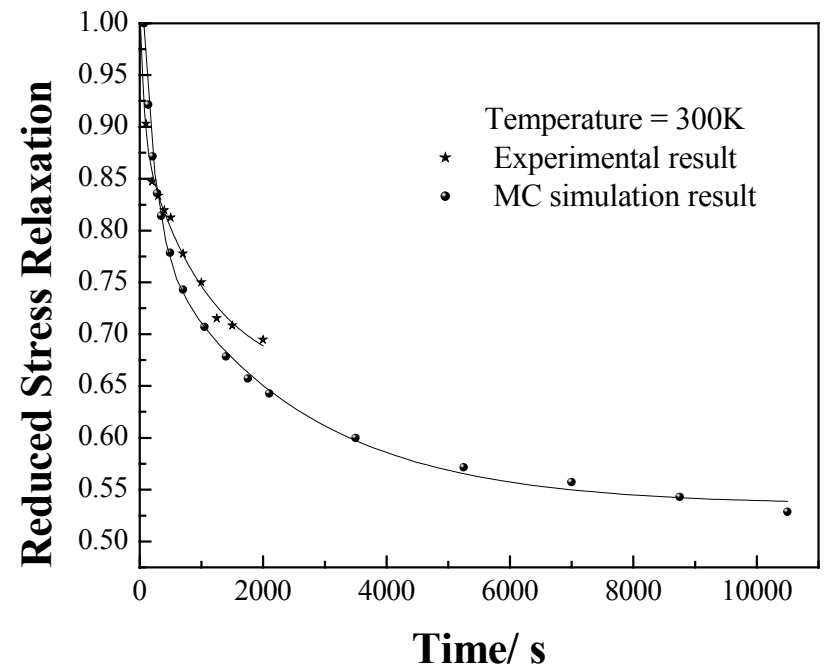

Fig. 6. MC simulation and experimental [23] stress relaxation for high-density polyethylene

The obtained results allow the suggestion that the method of MC together with the model and the employed dynamics give a satisfactory description of the mechanical properties and relaxation phenomena of polyethylene. The dynamics applied to the whole assembly of chains result in a deformation of the system without changing the initial bond length as it is expected to occur during the first deformation steps. A 
quantitative correlation of the theoretical and experimental graphs is possible since the deformation rates of the simulation are not very high (strain times in the order of $\mathrm{mcs} \approx 70 \mathrm{~s}$ ).

[1] Carmesin, I.; Kremer, K.; Macromolecules 1998, 21, 2819.

[2] Brostow, W.; Corneliussen, R. D.; "Failure of Plastics", Hanser, New York 1986.

[3] Akinay, A. E.; Brostow, W.; Castaño, V. M.; Maksimov, R.; Olszynski, P.; Polymer 2002, 43, 3593.

[4] Brostow, W.; Donahue, M.; Karashin, C. E.; Simoes, R.; Mat. Res. Innovat. 2001, 4,75

[5] Sharaf, M. A.; Kloczkowski, A.; Mark, J. E.; Comput. Theor. Polym. Sci. 2001, 11, 251.

[6] Golding, I.; Kantor, Y.; Phys. Rev. E. 1997, 56, 1318.

[7] Benneman, C.; Baschnagel, J.; Paul, W.; Binder, K.; Comput. Theor. Polym. Sci. 1999, 9, 21.

[8] Andrejew, E.; Baschnagel, J.; Phys. A 1996, 233,117.

[9] Zhang, L.; Xu, Y.; Zhao, D.; Eur. Polym. J. 2000, 36,1607.

[10] Tries, V.; Paul, W.; Baschnagel, J.; Binder, K.; J. Chem. Phys. 1996, 106, 738.

[11] Haire, K. R.; Carver, T. J.; Windle, A. H.; Comput. Theor. Polym. Sci. 2001, 11, 17.

[12] Binder, K.; Baschnagel, J.; Paul, W.; Wittmann, H. P.; Wolfgardt, M.; Comput. Mat. Sci. 1995, 4, 309.

[13] Ospina, S.; Restrepo, J.; López, B.; Mat. Res. Innovat. 2003, 7, 1.

[14] Ohno, K.; Esfarjani, K.; Kawazoe, Y.; "Computational Materials Science: From Ab Initio to Monte Carlo Methods", Springer-Verlag, Berlin 1999.

[15] Sista, S.; Debroy, T.; Metallurgic Mat. Trans. B 2001, 32B, 1195.

[16] Porter, D.; “Group Interaction Modelling of Polymer Properties”, Marcel Dekker Inc., 1995.

[17] Van Krevelen, D.; "Properties of Polymer", Elsevier Publishing Company, 1976.

[18] Newman, M. E. J.; Barkema, G. T.; "Monte Carlo Methods in Static Physics", Clarendon-Press, Oxford 1999.

[19] Gedde, U. W.; “Polymer Physics”, Chapman \& Hall, London 1995.

[20] Mischler, C.; Baschnagel, J.; Binder, K.; Cond-mat./ 0012277, 2000.

[21] Annual Book of ASTM Standards 2000, 08.01 West Conshohocken, PA.

[22] Shiro, Matsuoka; "Relaxation Phenomena in Polymers", Hanser Publishers, München 1992.

[23] Mansour, S. A.; Egypt. J. Sol. 2001, 24, 89. 